\title{
New fronts in an old war
}

\author{
There has been no new treatment for tuberculosis for three decades. But \\ there is now the potential for a radical resurgence of drug development, \\ says Declan Butler, if the political and industrial climate stays fair.
}

$\mathrm{N}$ ext time you are in a busy street, glance around. If you live in North America or Western Europe, one in ten of those walking past you is probably infected with Mycobacterium tuberculosis the bacterium that causes TB. And with infection rates in developing countries and Eastern Europe soaring, the global average is close to one in three.

Thankfully, the virulence of M. tuberculosis does not match its prevalence: less than a tenth of those infected will develop the fullblown disease. In the rest, the bacterium seems to lie dormant. But the number of cases where this latent TB becomes activated is increasing, mainly because the rise in HIV infection is weakening the immune systems of those also carrying M. tuberculosis. At the same time, many strains of the bacterium are developing resistance to the drugs used to treat the disease. Together, these trends have led the World Health Organization (WHO) to declare a global health emergency (see 'The rise and rise of tuberculosis', opposite).

"We need new drugs," concludes the draft of a scientific blueprint being prepared by the Global Alliance for TB Drug Development, a consortium of scientists, donor agencies and pharmaceutical companies set up earlier this year to provide a new impetus for the field ${ }^{1}$. The document will be released in the autumn.

The good news is that recent scientific developments, including the sequencing of the M. tuberculosis genome ${ }^{2}$, mean there is a chance of making significant progress. "This is the most exciting time for TB drug development in over two decades," says Rick O'Brien, chief of research in the Division of

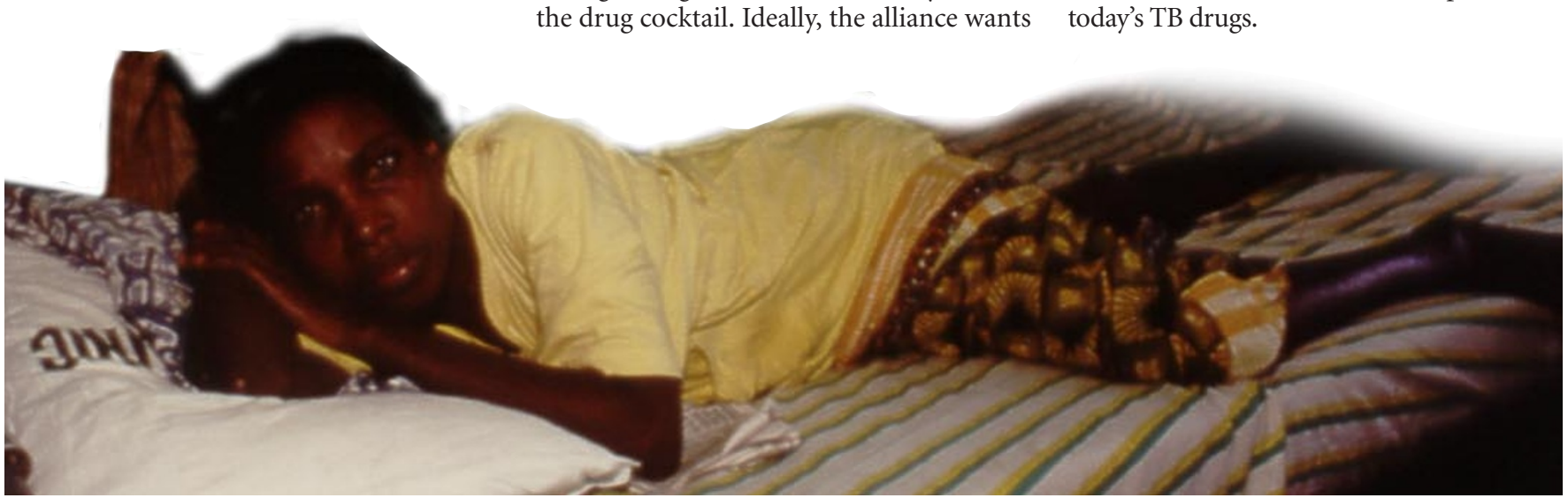

Kill or cure? More than one in three people in the developing world is infected with TB and unless new drugs are found to fight the disease, many will die.
TB Elimination at the Centers for Disease Control and Prevention in Atlanta, Georgia, and rapporteur for the alliance's scientific blueprint. "At long last technical advances and increasing interest in the global TB problem are coming together."

\section{Traditional treatment}

TB is usually treated with a cocktail of four drugs - ethambutol, isoniazid, pyrazinamide and rifampicin. These drugs are ineffective against latent TB, but work well when M. tuberculosis is actively dividing. Two months of treatment can reduce infection in sick patients to the point where they can no longer transmit the disease. But in the face of this pharmaceutical assault, the remaining bacteria slow their cell division and enter a persistent state that resembles latent infection. It can take another six months of treatment to kill these remaining bacteria.

For this reason, the WHO devised a scheme called DOTS, or Directly Observed Treatment, Short-course. In DOTS, doctors actively diagnose TB cases and subsequently ensure that patients swallow each and every pill - which may also include the broadspectrum antibiotic streptomycin - over six to eight months. But DOTS requires trained medical staff, infrastructure and money that lie beyond the resources of many developing countries. In practice, a quarter of TB cases are not diagnosed before they become infectious, and many people given the treatment do not stay the course.

The Global Alliance for TB Drug Development says its priority is to develop a drug that will speed up elimination of the slow-growing bacteria that initially survive the drug cocktail. Ideally, the alliance wants



The enemy within: Mycobacterium tuberculosis.

to reduce the total time needed for treatment to two months or less, says Ariel PablosMendez, associate director for health equity at the Rockefeller Foundation in New York. This would be a revolution in TB control, he adds.

\section{Persistent problem}

Given the similarities between the persistent bacteria and those involved in latent infections, such a drug might also prove effective in eradicating M. tuberculosis from asymptomatic TB-positive people. Many researchers agree that studying slow-growing $M$. tuberculosis is a top priority. "If you could find a drug that would kill these persistent organisms that would be an enormous step forward," says Denis Mitchison, now retired, but continuing to carry out research at $\mathrm{St}$ George's Hospital in London, where he worked on the clinical development of today's TB drugs. 
Alternatively, it might be possible to force the bacteria to start dividing rapidly again, so that other drugs could do their work. "We could prevent latency, or even stimulate persistent or dormant bacilli to grow and be better able to kill them," says William Bishai of Johns Hopkins University in Baltimore, Maryland.

New families of drugs that can target dividing $M$. tuberculosis are also urgently needed to sidestep growing bacterial resistance to isoniazid and rifampicin. Multipledrug-resistant TB accounts for around $2 \%$ of all cases worldwide, and this figure is increasing. The proportion of cases that are multidrug resistant has reached $14.1 \%$ in Estonia, and more than $25 \%$ in Russia's prisons.

The problem confronting drug developers is that decades of neglect in TB research - partly fostered by the misconception that existing drugs had beaten the disease - have left severe gaps in our knowledge of the biology of M. tuberculosis. "We know singularly little about pathogenesis," says Mitchison.

\section{Bright prospects}

But morale among TB researchers is improving. There is a raft of new scientific opportunities and $M$. tuberculosis has a host of unique features that, in principle, should make it a relatively easy target for drugs. In addition, there is renewed political and public interest worldwide in funding TB research. For example, at last month's summit in Okinawa, Japan, the G8 economic powers pledged to halve the number of $\mathrm{TB}$ deaths and the prevalence of disease by 2010 .

Much of the impetus for research has come from the availability of the M. tuberculosis genome. Functions have been identified for almost half of the bacterium's genes ${ }^{2}$, and genomic approaches are now being applied to drug discovery. At the Affymax Research Institute, a biotech company in Santa Clara, California, Mike Wilson is using microarrays of the bacterium's 4,000 known genes to investigate the effects of TB drugs on gene expression $^{3}$. By adding 'complementary' DNA, which is made from the bacterium's messenger RNA and binds to the corresponding genes on the microarray, he can see which genes are active before and during treatment.

Wilson is now working with Clifton Barry's group at the National Institute of Allergy and Infectious Diseases in Bethesda, Maryland, to speed up screening of candidate drugs by profiling gene expression patterns specific to particular biochemical pathways - starting with the production of M. tuberculosis's characteristic waxy cell wall. "These are complex structures that require many biosynthetic steps, so we might expect to find a variety of potentially useful targets if we could identify the set of genes responsible," says Wilson. Initially, they are concentrating on finding new families of drugs that have similar effects on gene expression to isoniazid, which is known to disrupt cell-wall formation.

But latent TB remains a mystery. 'Latent' is a clinical definition, meaning simply that the bacteria are present but there is no active disease. Biologists do not know whether the bacteria are lying dormant or are actively replicating but held in check by the immune system. "I prefer the term 'persistent and chronic TB infection'," says David Russell of Cornell University in Ithaca, New York. "The terms dormancy and latency imbue the bacterium with properties that have not really been demonstrated.'

\section{Cell in-mates}

One hypothesis is that the bacteria hide inside immune cells known as macrophages. These are large cells that ingest foreign microorganisms and cellular debris in diseased tissues. Unlike other bacteria, M. tuberculosis can survive inside these cells, where it initially grows and divides. Over time, the immune system walls off infected macrophages into structures called granulomas, and the bacteria slow down their division. There they can lie for many years before suddenly resuming their rapid multiplication and bursting out of the granuloma to cause active disease.

Inside a granuloma, $M$. tuberculosis has to adapt to declining levels of oxygen. To investigate this, Lawrence Wayne of the Long Beach Veterans Affairs Medical Center in California has devised an experimental model in which oxygen levels in M. tuberculosis cultures are gradually reduced so that the bacteria survive but do not divide ${ }^{4}$.

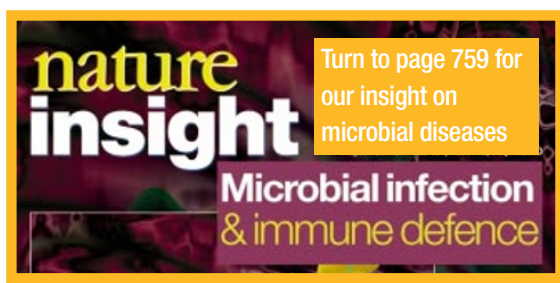

Other researchers are studying infected mice which develop a chronic infection similar to latent human TB. But mouse granulomas are different from those seen in humans - they do not form structures that can eventually rupture into a bronchus, allowing virulent bacteria to be coughed out. Guinea pigs $^{5}$ and rabbits ${ }^{6}$ provide better models, but it is more difficult to obtain large inbred populations and, unlike mice, these animals can spread the disease to humans. As a result, they can only be studied in expensive isolation facilities.

\section{Drugs on target}

Although all of the models have shortcomings, they have helped to identify many new drug targets for persistent and chronic forms of M. tuberculosis over the past year or so. Among the most exciting is the discovery, announced in this issue of Nature ${ }^{7}$ by a team led by Russell, that an enzyme called isocitrate lyase seems to be crucial to the survival of M. tuberculosis in granulomas. This enzyme allows the bacteria to get energy and build carbohydrates from fatty acids in the absence of oxygen.

Russell and his colleagues, who this month also reported the crystalline struc-

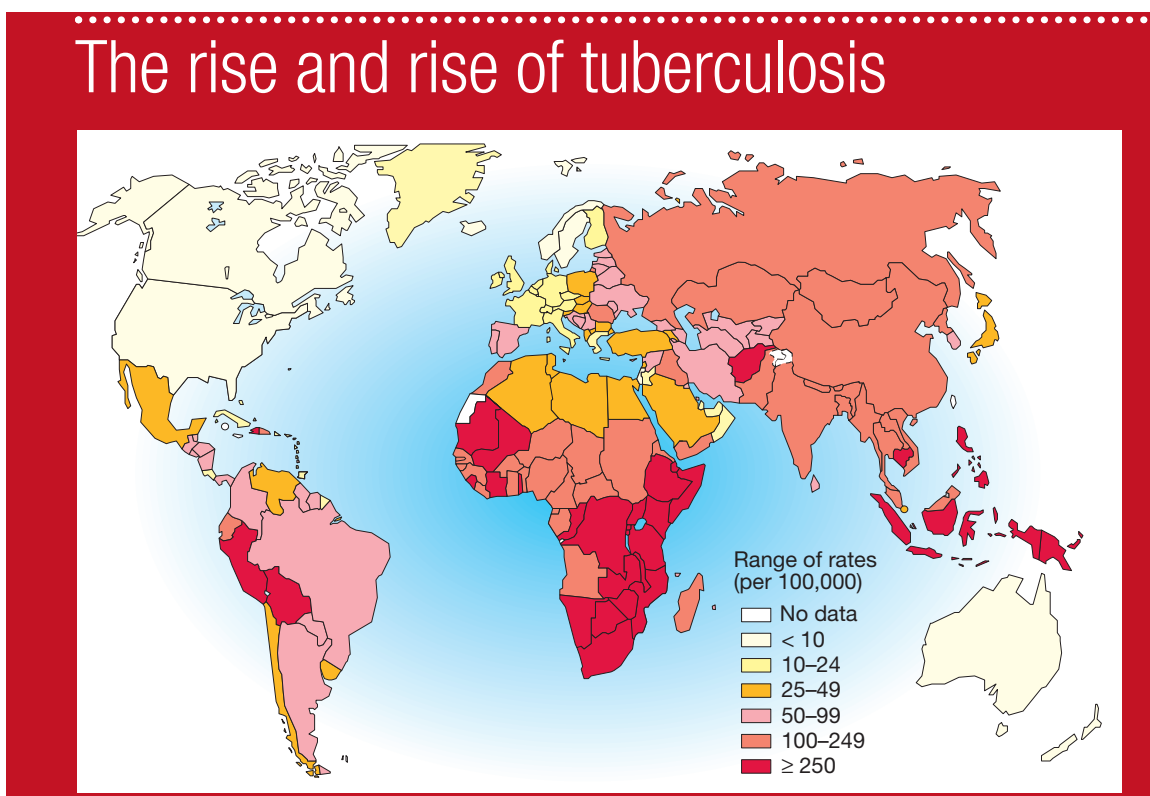

Cases of active TB per 100,000 population for 1997 (source: ref. 13).

- 8 million new cases of active TB annually, including 3.5 million cases of infectious pulmonary disease - 2 million of these $-5,000$ per day - will die - 16 million existing cases of active TB
- Without new control measures, one billion new cases of TB are predicted between now and 2020, of whom 200 million will develop active disease, and 35 million will die. Annual incidence of new cases will grow to 11 million 
ture of isocitrate lyase ${ }^{8}$, are now working to develop inhibitors of the enzyme. The UKbased drug company Glaxo Wellcome is also taking an interest. "We have already begun to look for inhibitors of the isocitrate lyase enzyme in our library of compounds, using the structure of the protein to guide the search," says Ken Duncan, manager of Glaxo Wellcome's Action TB programme.

Another clue to the genes involved in persistent infection came earlier this year from Stanley Falkow's laboratory at Stanford University in California. Falkow and his colleagues studied M. marinum, which causes latent TB in frogs and is the closest relative of M. tuberculosis. They identified gene promoters that were only switched on when the bacteria were encapsulated in frog granulomas. Two of these were from genes in the PE-PGRS family, and when the researchers examined the sequence of the genomic region containing these genes they found a third family member'. PE-PGRS genes code for unusual proteins that are rich in the amino acid glycine but have unknown function. They are unique to the genus Mycobacterium and account for more than $10 \%$ of the coding regions of its genome ${ }^{2}$.

Falkow and his colleagues created strains of M. marinum in which each of the three genes was knocked out individually. Two of these knockout strains could not divide in macrophages, and one showed greatly reduced persistence in frog granulomas. The group now intends to knock out all three genes in unison to see whether this will reduce the bacterium's persistence further. The researchers hope that similar genes in $M$. tuberculosis will play the same role in human TB, and therefore make useful drug targets. "We know that these genes have a role in virulence," says Lalita Ramakrishnan, a member of the team.

Hot on the heels of Falkow's paper came the news, from a group led by Kendall Stover

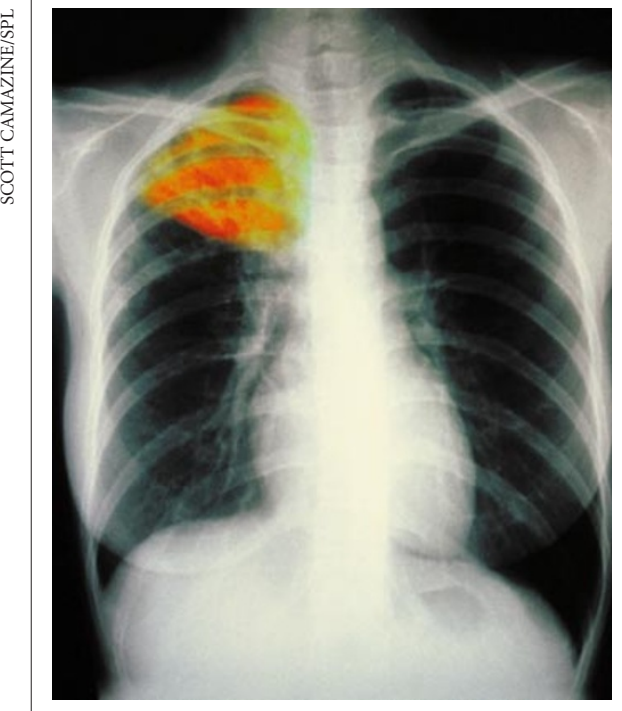

Shadow of death: TB lesion in the lung. at PathoGenesis, a company in Seattle, that molecules called nitroimidazopyrans seem to kill M. tuberculosis, including persistent, slowdividing bacteria ${ }^{10}$. This discovery stemmed from observations that related molecules called bicyclic nitroimidazofurans, originally developed to improve the effectiveness of cancer radiotherapy, were active against M. tuberculosis. Those drugs were too mutagenic to use against TB, however.

\section{Survival strategies}

Meanwhile, researchers led by Jean Pieters at the Basel Institute for Immunology in Switzerland have been looking at how $M$. tuberculosis bacteria living inside macrophages avoid attack by lysosomes cell structures containing enzymes that break down bacteria and debris ingested by the macrophages. Last year, they discovered that a macrophage protein called tryptophane aspartate-containing coat protein, or TACO, seems to play a key role ${ }^{11}$. When the bacteria are ingested by a macrophage they end up surrounded by a host-cell membrane, forming a structure called a phagosome. The researchers found that TACO surrounds these phagosomes, preventing transport of the bacteria to the lysosomes.

Pieters wanted to discover how to release TACO from the phagosomes. After testing several chemicals, he found that digitonin, a solubilizing agent, completely released the protein $^{12}$. It occurred to Pieters and his colleague John Gatfield that digitonin also binds to cholesterol. "As we knew TACO was responsible for mycobacterial survival within phagosomes, we reasoned that if we get rid of the cholesterol, TACO cannot be recruited to the phagosome and the mycobacteria will be transferred to lysosomes and killed," says Pieters.

When the researchers tested this idea in cultures of macrophages, they found that reducing cholesterol had a second, even more dramatic effect. "To our surprise we found that the bacteria were no longer taken up by the macrophages," says Pieters. "Cholesterol thus has dual roles: getting the bacteria to be taken up, and then conferring protection from lysosomes."

It would not be feasible to reduce cholesterol levels as a therapy for persistent TB. But Pieters believes it might be possible to identify cholesterol-binding sites on M. tuberculosis, block them with drugs and so prevent the bacteria entering macrophages. He and his colleagues are now planning to look at mutant bacteria that cannot get into macrophages to work out which genes are involved.

TB researchers are optimistic that continued research will generate many more potential drug targets. But the route from a target to a drug is long and expensive, and the big unknown is whether drug companies will invest the multimillions of dollars needed for
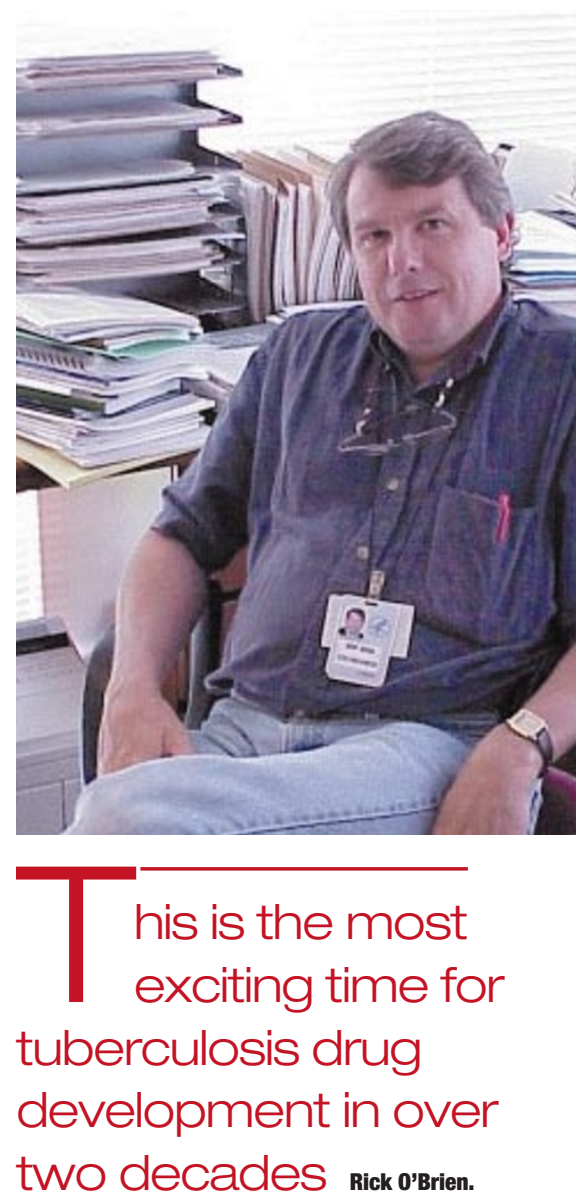

drug development. Realizing this, the Global Alliance for TB Drug Development is preparing a report on 'pharmaco-economics' alongside its scientific blueprint. This will assess in detail the size of the TB drug market, current investment and gaps in corporate research and development. It plans to use this report as the basis for a business plan for a joint drug-discovery programme between the public and private sectors.

The alliance's stated goal is to register one new drug for chronic and persistent TB by 2007 , and a second by 2012 . With the difference between success and failure being measured in millions of human lives, the stakes could not be much higher.

Declan Butler is Nature's European correspondent.

1. Butler, D. Nature 403, 692 (2000)

2. Coles, S. T. et al. Nature 393, 537-544 (1998).

3. Wilson, M. et al. Proc. Natl Acad. Sci. USA 96, 12833-12838 (1999).

4. Wayne, L. G. \& Haynes, L. G. Infect. Immun. 64, 2062-2069 (1996)

5. O'Grady, F. \& Riley, R. L. Adv. Tuberc. Res. 12, 150-190 (1963).

6. Dannenberg, A. M. in Tuberculosis: Pathogenesis, Protection and Control (ed. Bloom, B. R.) 149-156 (American Society for Microbiology, Washington DC, 1994).

McKinney, J. D. et al. Nature 406, 735-738 (2000).

8. Sharma, V. et al. Nature Struct. Biol. 7, 663-668 (2000).

9. Ramakrishnan, L., Federspiel, N. A. \& Falkow, S. Science 288, 1436-1439 (2000).

10. Stover, C. K. et al. Nature 405, 962-966 (2000).

11. Ferrari, G., Naito, N. H., Langen, J. \& Pieters, J. Cell 97 , 435-447 (1999).

12. Gatfield, J. \& Pieters, J. Science 288, 1647-1651 (2000). 13. Dye, C., Scheele, S., Dolin, P., Pathania, V. \& Raviglione, M. C. J. Am. Med. Assoc. 282, 677-686 (1999) 